\title{
ON SECTIONAL CURVATURES AND CHARACTERISTIC OF HOMOGENEOUS SPACES
}

\author{
WERNER GREUB AND PHILLIPPE TONDEUR ${ }^{1}$
}

Let $X$ be a compact orientable Riemannian manifold of even dimension $n$. The generalized Gauss-Bonnet theorem [1] states that the Euler-Poincare characteristic of $X$ is

$$
\chi(X)=\frac{2}{c_{n}} \int_{X} \gamma_{n} \omega
$$

where $c_{n}$ is the volume of the Euclidean unit $n$-sphere, $\gamma_{n}$ the $n$th sectional curvature (see the definition (2) below) and $\omega$ the volume element of the Riemannian structure of $X$. It is a still open question, whether the fact that the usual sectional curvature (second order sectional curvature) $\gamma_{2}$ has a constant sign for all plane sections, has some implications on the sign of $\gamma_{n}$. Such results would give interesting applications via the generalized Gauss-Bonnet theorem. A known result in this direction is Milnor's theorem (see [2, Theorem 5]), stating that for $n=4$ the Euler-Poincaré characteristic is positive, if $\gamma_{2}$ is always positive or always negative.

We shall consider the class of Riemannian manifolds arising by division of a compact Lie group $G$ by a closed subgroup $H$ and equipment of the quotient $G / H$ with the invariant Riemannian metric $g$ induced by a bi-invariant metric $\tilde{g}$ on $G$. Consider the orthogonal decomposition

$$
G=H \oplus M
$$

with respect to $g$, turning $G / H$ in to a reductive homogeneous space. We shall make the assumption that $G / H$ is locally symmetric, i.e. $[M, M] \subset H$. Let $n=\operatorname{dim} G / H$. With these notations we shall prove the following.

THEOREM. Let $p$ be any even integer with $0<p \leqq n$. Then the pth sectional curvature $\gamma_{p}$ is nonnegative.

REMARK. For $p=2$ this was proved in [6] even without the assumption of the local symmetry of $G / H$, and follows also at once from the formulae in [5]. As remarked in [7], the proof of Milnor's result in [2] shows that $\gamma_{2} \geqq 0$ implies $\gamma_{4} \geqq 0$.

Received by the editors February 10, 1965. sity.

1 The second author was supported by NSF grant GP-1217 at Harvard Univer- 
Corollary [3]. Let the situation be as before. Then the EulerPoincaré characteristic of $G / H$ is nonnegative.

Proof. Without loss of generality we can assume that $H$ is connected. Hence $G / H$ is orientable. We only have to consider the case when the dimension $n$ of $G / H$ is even. Then the Gauss-Bonnet theorem yields the desired result.

This answers, for the class of spaces considered, the question raised in [6, p. 13, line 5].

Remark. Suppose the dimension $n$ of $G / H$ to be even. Then by homogeneity, the sectional curvature $\gamma_{n}$ is seen to be constant [7]. Therefore $\chi(G / H)$ and $\gamma_{n}$ are either both positive or both zero.

We recall the definition of the sectional curvatures of a Riemannian manifold $X$ of (not necessarily even) dimension $n$ (see [7]). Let $p$ be an even integer with $0<p \leqq n, x \in X$ and $P \subset T_{x}(X)$ a $p$-plane at $x$. Let $X_{1}, \cdots, X_{p}$ be any orthonormal base of $P$ and $R$ the curvature tensor at $X$ of the Riemannian metric $\langle$,$\rangle . Then the p$ th sectional curvature of the $p$-plane $P$ is given by

$$
\begin{aligned}
\gamma_{p}(x ; P)=\frac{(-1)^{P-2}}{2^{p / 2} \cdot p !} \sum_{\sigma, \tau} \epsilon(\sigma) \epsilon(\tau)\left\langle R\left(X_{\sigma_{1}}, X_{\sigma_{2}}\right) X_{\tau_{1}}, X_{\tau_{2}}\right\rangle \cdots \\
\cdots\left\langle R\left(X_{\sigma_{p-1}}, X_{\sigma_{p}}\right) X_{\tau_{p-1}}, X_{\tau_{p}}\right\rangle .
\end{aligned}
$$

Here the sum ranges over all permutations $\sigma, \tau$ of the set $\{1, \cdots, p\}$ and $\epsilon(\sigma), \epsilon(\tau)$ are the signs of the permutations $\sigma, \tau$ respectively. For $p=2$, formula (2) is the usual expression

$$
\gamma_{2}(x ; P)=-\left\langle R\left(X_{1}, X_{2}\right) X_{1}, X_{2}\right\rangle
$$

in view of the skew-symmetry of the operator $R\left(X_{1}, X_{2}\right)$ with respect to $\langle$,$\rangle .$

We now turn to the case $X=G / H$ considered in the theorem. As $G / H$ is supposed to be locally symmetric in its canonical reductive structure, the canonical connection [5] is the Riemannian connection of $\tilde{g}$. Let $\langle\rangle:, G \times G \rightarrow R$ denote the restriction of $g$ to $G$ and let $x_{0}$ be the point of $G / H$ corresponding to the unit $e$ of $G$. $T_{x_{0}}(G / H)$ is identified with the orthogonal complement $M$ of $H$ in $G$. Then we have the following known

Lemma. Let $R$ be the curvature tensor of $\tilde{g}$ in $x_{0}$. Then for $X, Y, V$, $W \in T_{x_{0}}(G / H)$ we have

$$
\langle R(X, Y) V, W\rangle=-\langle[X, Y],[V, W]\rangle .
$$

Proof. By [5, Theorem 10.3], we have in view of the preceding remarks 


$$
R(X, Y) V=-[[X, Y], V] .
$$

Note that $[X, Y] \in H$, as we have supposed $G / H$ to be locally symmetric. Now $\langle$,$\rangle is invariant under the adjoint representation of H$, so that

$$
\langle[[X, Y], V], W\rangle=\langle[X, Y],[V, W]\rangle .
$$

This proves the lemma.

The theorem is now a consequence of the following

Proposition. Let the situation be as above. Let $P \subset T_{x_{0}}(M)$ be a $p$-plane, $p$ an even integer with $0<p \leqq n=\operatorname{dim}(G / H)$ and $X_{1}, \cdots, X_{r}$ $(r=\operatorname{dim} G)$ an orthonormal basis of $G$ such that the first $p$ vectors lie in $P$ and the last $r-n$ vectors in $H$. Then the pth sectional curvature is given by

$$
\begin{aligned}
& \gamma_{p}\left(x_{0} ; P\right)=\frac{1}{2^{p / 2} \cdot p !} \\
& \cdot \sum_{k_{1}, \cdots, k_{p} / 2}\left(\sum_{\sigma} \epsilon(\sigma)\left\langle\left[X_{\sigma_{1}}, X_{\sigma_{2}}\right], X_{k_{1}}\right\rangle \cdots\left\langle\left[X_{\sigma_{p-1}}, X_{\sigma_{p}}\right], X_{k_{p} / 2}\right\rangle\right)^{2}
\end{aligned}
$$

where $\sigma$ runs through the permutations of $\{1, \cdots, p\}, \epsilon(\sigma)$ is the sign of $\sigma$, and $\left(k_{1}, \cdots, k_{p / 2}\right)$ runs through the $p / 2$-tuples of integers $k_{i}$ with $r-n<k_{i} \leqq r$ for $i=1, \cdots, p / 2$.

Proof. By (2), we have in virtue of the lemma

$$
\begin{array}{r}
\gamma_{p}\left(x_{0} ; P\right)=\frac{1}{2^{p / 2} \cdot p !} \sum_{\sigma, \tau} \epsilon(\sigma) \epsilon(\tau)\left\langle\left[X_{\sigma_{1}}, X_{\sigma_{2}}\right],\left[X_{\tau_{1}}, X_{\tau_{2}}\right]\right\rangle \cdots \\
\cdots\left\langle\left[X_{\sigma_{p-1}}, X_{\sigma_{p}}\right]\left[X_{\tau_{p-1}}, X_{\tau_{p}}\right]\right\rangle .
\end{array}
$$

We write $c_{\alpha \beta}^{\gamma}$ for $\left\langle\left[X_{\alpha}, X_{\beta}\right], X_{\gamma}\right\rangle$, so that $\left[X_{\alpha}, X_{\beta}\right]=\sum_{\gamma=1}^{r} c_{\alpha \beta}^{\gamma} X_{\gamma}$ (the $c$ 's are the structural constants of $G)$. Using $[M, M] \subset H$ and the orthonormality of the base we obtain then

$$
\left\langle\left[X_{\sigma_{1}}, X_{\sigma_{2}}\right],\left[X_{\tau_{1}}, X_{\tau_{2}}\right]\right\rangle=\sum_{k_{1}=r-n+1}^{r} \begin{gathered}
k_{\sigma_{1}} \\
c_{1} \sigma_{2} \\
c_{\tau_{1} \tau_{2}}
\end{gathered}
$$

and similar expressions for the other terms in (5). Hence

$$
\begin{aligned}
& \gamma_{p}\left(x_{0} ; P\right)=\frac{1}{2^{p / 2} \cdot p !} \sum_{\sigma, \tau} \epsilon(\sigma) \epsilon(\tau)\left(\sum_{k_{1}=r-n+1}^{r} \begin{array}{cc}
k_{1} & k_{1} \\
c_{\sigma_{1} \sigma_{2}} c_{\tau_{1} \tau_{2}}
\end{array}\right) \cdots \\
& \cdots\left(\sum_{k_{p} / 2=r-n+1}^{r} \quad c_{\sigma_{p-1} \sigma_{p}}^{k_{p} / 2} c_{\tau_{p-1} / 2}^{k_{p}}\right) \text {. }
\end{aligned}
$$


The $\sum_{\sigma, r}$ in (6) can be rewritten as

$$
\sum_{k_{1}, \cdots, k_{p} / 2=r-n+1}^{\tau}\left(\sum_{\sigma} \epsilon(\sigma) c_{\sigma_{1} \sigma_{2}}^{k_{1}} \cdots c_{\sigma_{p-1} \sigma_{p}}^{k_{p} / 2}\right)\left(\sum_{\tau} \epsilon(\tau) c_{\tau_{1} \tau_{2}}^{k_{1}} \cdots c_{\tau_{p-1} \tau_{p}}^{k_{p} / 2}\right)
$$

or

$$
\sum_{k_{1}, \ldots, k_{p} / 2=r-n+1}^{r}\left(\sum_{\sigma} \epsilon(\sigma) c_{\sigma_{1}}^{k_{1}} c_{\sigma_{2}} \cdots c_{\sigma_{p-1} \sigma_{p}}^{k_{p / 2}}\right)^{2} .
$$

This proves the proposition.

Observe that for the usual sectional curvature $\gamma_{2}$ one obtains (directly by the lemma) the expression

$$
\gamma_{2}\left(x_{0} ; P\right)=\left\langle\left[X_{1}, X_{2}\right],\left[X_{1}, X_{2}\right]\right\rangle
$$

i.e. $\gamma_{2}\left(x_{0} ; P\right)=0$ if and only if $\left[X_{1}, X_{2}\right]=0$ (see [6]). Thus by (4) we clearly have the implication $\gamma_{2}=0 \Rightarrow \gamma_{p}=0$; for all even $p$ with $0<p \leqq n$. This is true for any Riemannian manifold [7, Theorem 6.4].

We remark that our theorem applies in particular to compact Riemannian symmetric spaces equipped with the metric arising naturally from a bi-invariant metric on the group of isometries.

The manifold of a compact Lie group $G$ is with respect to a biinvariant metric $g$ a Riemannian symmetric space and one can obtain the sectional curvatures by applying the proposition. But in this case it is simpler to observe that the (0)-connection of $G[5$, p. 49] is the Riemannian connection of $g$. The expression $R(X, Y) V=$ $-[[X, Y], V] / 4$ for the curvature tensor $R$ in $e ; X, Y, V \in G$ $[5$, p. 49] shows that

$$
\langle R(X, Y) V, W\rangle=-\langle[X, Y],[V, W]\rangle / 4 .
$$

By a similar computation as in the proof of the proposition, one obtains for the $p$ th sectional curvature $\gamma_{p}$ on a $p$-plane $P \subset G$ the expression

$$
\begin{aligned}
& \gamma_{p}(e ; P)=\frac{1}{2^{3 p / 2} \cdot p !} \\
& \cdot \sum_{k_{1}, \cdots, k_{p} / 2}\left(\sum_{\sigma} \epsilon(\sigma)\left\langle\left[X_{\sigma_{1}}, X_{\sigma_{2}}\right], X_{k_{1}}\right\rangle \cdots\left\langle\left[X_{\sigma_{p}-1}, X_{\sigma_{p}}\right], X_{k_{p} / 2}\right\rangle\right)^{2}
\end{aligned}
$$

where $X_{1}, \cdots, X_{n}$ is an orthonormal base of $G, \sigma$ runs through the permutations of $\{1, \cdots, p\}$ and $\left(k_{1}, \cdots, k_{p / 2}\right)$ runs now through all $p / 2$-tuples of integers $k_{i}$ with $1 \leqq k_{i} \leqq n$ for $i=1, \cdots, p / 2$.

For an even-dimensional group it is clear that $\gamma_{n}=0$ by the generalized Gauss-Bonnet theorem. (7) gives therefore the identity 


$$
\sum_{\sigma} \epsilon(\sigma)\left\langle\left[X_{\sigma_{1}}, X_{\sigma_{2}}\right], X_{k_{1}}\right\rangle \cdots\left\langle\left[X_{\sigma_{n-1}}, X_{\sigma_{n}}\right], X_{k_{n} / 2}\right\rangle=0
$$

valid for any orthonormal base $X_{1}, \cdots, X_{n}$ of $G$ and any $n / 2$-tuple of integers $k_{i}$ with $1 \leqq k_{i} \leqq n$ for $i=1, \cdots, n / 2$.

\section{REFERENCES}

1. S. S. Chern, $A$ simple intrinsic proof of the Gauss-Bonnet theorem for closed Riemannian manifolds, Ann. of Math. 45 (1944), 747-752.

2. - On curvature and characteristic classes of a Riemannian manifold, Abh. Math. Sem. Univ. Hamburg 20 (1956), 117-126.

3. H. Hopf and H. Samelson, Ein Satz über die Wirkungsräume geschlossener Liescher Gruppen, Comment. Math. Helv. 13 (1940-1941), 240-251.

4. A. Lichnerowicz, Géométrie des groupes de transformations, Dunod, Paris, 1958.

5. K. Nomizu, Invariant affine connexions on homogeneous spaces, Amer. J. Math. 76 (1954), 33-65.

6. H. Samelson, On curvature and characteristic of homogeneous spaces, Michigan Math J. 5 (1958), 13-18.

7. J. Thorpe, Sectional curvatures and characteristic classes, Ann. of Math. 80 (1964), 429-443.

UNIVERSITY OF TORONTO,

HARVARD UNIVERSITY AND

University of CALIFornia, Berkeley 